\title{
Actuarial Pricing Models of Reverse Mortgage with the Stochastic Interest Rate
}

\author{
N.N. Jia, H. Yang \& J.B. Yang \\ College of Science, Harbin Engineering University, Harbin, 150001, China

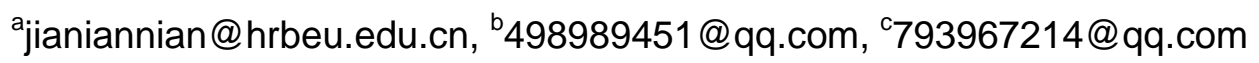

Keywords: Reverse mortgage; Actuarial pricing; Stochastic interest rate

\begin{abstract}
The aging problem became more important and serious in China in recent years. The reverse mortgage is an innovation model to support the aged people. In this study, the accumulation function model of interest force with a Wiener process and a negative-binomial distribution is proposed as the basis for the reverse mortgage. With the proposed model, a lump sum pricing model, annuity pricing model, linear increasing annuity pricing model for single-life and double-lives are provided. All of the models can be improved to solve the problem that the actuarial pricing models of reverse mortgage only could be calculated by the fixed interest rate.
\end{abstract}

\section{Introduction}

Lending rate in traditional reverse mortgages actuarial pricing model is assumed to be fixed. However, in real life loan interest rate is easily affected by the surrounding environment, especially economic and policy factors, that is random. Therefore, building actuarial pricing models of reverse mortgage with the stochastic interest rate are necessary. Reed R, Gibler K.M. (2003) and Deokho C. studied the reverse mortgage loan of Australia and South Korea market demand [1-2]. Junfang Xi (2007) established a series of actuarial pricing models, and applied the option theory in the reverse mortgage loan pricing model [3]. Jinzhuo Li (2010) analyzed the actual needs of the elderly, and established a series of pricing models to measure the impact of price fluctuations on the reverse mortgage loan pricing [3]. This paper mainly studied models of reverse mortgage with the stochastic interest rate.

\section{Model framework and assumptions}

\section{Symbol description}

$\overline{L S^{\prime}}$ : The amount of the loan which is got by one borrower with a method of payment with the stochastic interest rate.

$\overline{P M T^{\prime}}$ : The amount of the loan which is got by one borrower at the beginning of each year with the method of annuity under the stochastic interest rate.

$\overline{P M T A^{\prime}}$ : The amount of the loan which is got by one borrower at the beginning of the first year by linear increasing annuity with the stochastic interest rate.

Q: The increasing amount every year of the loan when get the loan by linear increasing annuity.

$\mathrm{d}$ : The increasing ratio every year of the loan when get the loan by equal ratio increasing annuity.

$\overline{L S}^{\prime} \overline{x y}$ : The amount of the loan which is got by two borrowers by a sum of loan ceiling with the stochastic interest rate.

$\overline{P M T}_{x y}$ : The amount of the loan which is got by two borrowers at the beginning of each year by annuity with the stochastic interest rate.

$\overline{P M T A}_{x y}^{\prime}$ : The amount of the loan which is got by two borrowers at the beginning of the first year by linear increasing annuity with the stochastic interest rate.

\section{Model assumptions}

In order to simplify the analysis, the main assumptions of the pricing model are: (1)The borrower who applies for housing reverse mortgage apply for loan without taking the house as other mortgage. (2)The residual life of the borrower obey the distribution function of $\mathrm{T}(\mathrm{x})$. (3)The house is sold at 
market price immediately after the borrower died, without time gap between them. (4)Although the actual lifespan of the borrower can't be predicted in advance, if the amount of the people who apply for reverse mortgage is enough, we can ensure the death ratio every year of the borrower after signing the contract by the law of large numbers, we can inquire the newly revised life -expectancy tables, and we can size up the average remaining lifetime of the borrower. (5)The housing annual depreciation rate is obtained by the straight-line method, it already contains the postage of loan during the calculation, without setting other parameters. (6)In Two-life model, residual life T(x) and T(y) are independent of each other [4-5].

\section{The models of accumulated function of interest force with the stochastic interest rate}

In this section, we consider standard Wiener process and Negative-binomial distribution to build the accumulated function of interest force. The accumulation function of interest force can be specified as follows:

$$
R(t)=\delta t+\beta W(t)+\gamma N(t)
$$

Where $W(t)$ refer to standard Wiener process, where $N(t)$ defer to Negative-binomial distribution, $N(t) \sim N B(\alpha, P), W(t)$ and $N(t)$ are independent, $\delta$ is constant interest force, $\delta$ and $\gamma$ are constants, $\beta, \gamma \geq 0$. Lemmas are acquired as follows:

Lemma 2.1 If $W(t)$ follows standard Wiener process, that is $W(t) \sim N(0, t), \beta$ is constant, $t>0$, then

$$
E_{W}\left(e^{-\beta W(t)}\right)=e^{\frac{1}{2} \beta^{2} t} .
$$

Lemma 2.2 If $N(t) \sim N B(\alpha, P), \gamma$ is constant, $q=1-p$, then $E\left(e^{-\gamma N(t)}\right)=\left(\frac{p}{1-q e^{-\gamma}}\right)^{\alpha}$.

Lemma 2.3 If the accumulation function of interest rate is $R(t)=\delta t+\beta W(t)+\gamma N(t)$, discount function

is $v(t)=e^{-R(t)}=e^{-\delta t-\beta W(t)-\gamma N(t)}$, then its expectation is given by $E_{W} E_{N}(v(t))=e^{-\delta t+\frac{1}{2} \beta^{2} t}\left(\frac{p}{1-q e^{-\gamma}}\right)^{\alpha}$.

Where $W(t)$ follows standard Wiener process; $N(t) \sim N B(\alpha, p)$; $W(t)$ and $N(t)$ are independent; $\delta$ is constant interest force, $\delta$ and $\gamma$ are constants, $\beta, \gamma \geq 0$.

\section{Actuarial pricing model of single-live with the stochastic interest rate}

\section{Payment of single-live pricing model with the stochastic interest rate}

The present value of the housing property values at time $\mathrm{T}$ before the contract starts:

$$
L S(t)=H_{0}(1-h)^{t}(1+g)^{t} v(t)=H_{0}(1-h)^{t}(1+g)^{t} e^{-\delta t-\beta W(t)-\gamma N(t)}
$$

At the beginning of contract, the financing institutions pay the borrower a sum of loan ceiling. The loan ceiling equals to the present value of revenue which financing institutions auctioned houses immediately.

$$
\begin{aligned}
\overline{L S^{\prime}} & =E(L S(t))=E_{W} E_{N} E_{T}(L S(t))=E_{W} E_{N}\left(\int_{0}^{+\infty} L S(t)_{t} p_{x} \mu_{x+t} d t\right) \\
& =\int_{0}^{+\infty} H_{0}(1-h)^{t}(1+g)^{t} E_{W} E_{N}\left(e^{-\delta t-\beta W(t)-\gamma N(t)}\right)_{t} p_{x} \mu_{x+t} d t
\end{aligned}
$$

According to lemma 2.3, one single borrower who $x$ years old applied for reverse mortgage. With the stochastic interest rate, the payment of loan amount is given by

$$
\begin{aligned}
\overline{L S^{\prime}} & =\int_{0}^{+\infty} H_{0}(1-h)^{t}(1+g)^{t} e^{-\delta t+\frac{1}{2} \beta^{2} t}\left(\frac{p}{1-q e^{-\gamma}}\right)^{\alpha}{ }_{t} p_{x} \mu_{x+t} d t \\
& =\int_{0}^{\omega-x} H_{0}(1-h)^{t}(1+g)^{t} e^{-\delta t+\frac{1}{2} \beta^{2} t}\left(\frac{p}{1-q e^{-\gamma}}\right)^{\alpha}{ }_{t} p_{x} \mu_{x+t} d t
\end{aligned}
$$

\section{Annuity pricing model of single-live with the stochastic interest rate}

After the borrowers signed a loan contract with financing institutions, they get an equal loan from financing institutions until the borrowers die. The discounted value of the annuity equals to Eq. (7), then the loan amount of annuity is given by 


$$
\overline{L S^{\prime}}=E_{W} E_{N} E_{T}\left(\sum_{k=0}^{+\infty} \overline{P M T^{\prime}} I_{\{T>k\}} e^{-R(k)}\right)=\overline{P M T^{\prime}} E_{W} E_{N} E_{T}\left(\sum_{k=0}^{+\infty} I_{\{T>k\}} e^{-R(k)}\right)
$$

According to lemma 2.3, one single borrower who is $x$ years old applies for reverse mortgage. With the stochastic interest rate, the beginning of every year the payment of loan amount is given by

$$
\begin{aligned}
\overline{P M T^{\prime}} & =\frac{\overline{L S^{\prime}}}{E_{W} E_{N} E_{T}\left(\sum_{k=0}^{+\infty} I_{\{T>k\}} e^{-R(k)}\right)}=\frac{\overline{L S^{\prime}}}{\sum_{k=0}^{+\infty}{ }_{k} p_{x} E_{W} E_{N}\left(e^{-\delta k-\beta W(k)-\gamma N(k)}\right)} \\
& =\frac{\overline{L S^{\prime}}}{\sum_{k=0}^{\omega-x-1}{ }_{k} p_{x} e^{-\delta k+\frac{1}{2} \beta^{2} k}\left(\frac{p}{1-q e^{-\gamma}}\right)^{\alpha}}=\frac{\int_{0}^{\omega-x} H_{0}(1-h)^{t}(1+g)^{t} e^{-\delta t+\frac{1}{2} \beta^{2} t}{ }_{t} p_{x} \mu_{x+t} d t}{\sum_{k=0}^{\omega-x-1} e^{-\delta k+\frac{1}{2} \beta^{2} k}{ }_{k} p_{x}}
\end{aligned}
$$

\section{Linear increasing annuity pricing model of single-live with the stochastic interest rate}

If the borrower gets the loan which is increasing by a fixed amount Q every year, the actuarial present value of annuity is equal to total amount of payment.

$$
\begin{aligned}
\overline{L S^{\prime}} & =E_{W} E_{N} E_{T}\left(\sum_{k=0}^{+\infty}\left(\overline{P M T A^{\prime}}+k Q\right) I_{\{T>k\}} e^{-R(k)}\right) \\
& \left.=E_{W} E_{N} E_{T}\left(\sum_{k=0}^{+\infty} \overline{P M T A^{\prime}} I_{\{T>k\}} e^{-R(k)}\right)+E_{W} E_{N} E_{T} \sum_{k=0}^{+\infty} k Q I_{\{T>k\}} e^{-R(k)}\right) \\
& \left.=\overline{P M T A^{\prime}} E_{W} E_{N} E_{T}\left(\sum_{k=0}^{+\infty} I_{\{T>k\}} e^{-R(k)}\right)+Q E_{W} E_{N} E_{T} \sum_{k=0}^{+\infty} k I_{\{T>k\}} e^{-R(k)}\right)
\end{aligned}
$$

According to lemma 2.3, one single borrower who is $x$ years old applies for reverse mortgage by linear increasing annuity. With the stochastic interest rate, the beginning of the first year the payment of loan amount is given by

$$
\overline{P M T A^{\prime}}=\frac{\left.\overline{L S^{\prime}}-Q E_{W} E_{N} E_{T} \sum_{k=0}^{+\infty} k I_{\{T>k\}} e^{-R(k)}\right)}{E_{W} E_{N} E_{T}\left(\sum_{k=0}^{+\infty} I_{\{T>k\}} e^{-R(k)}\right)}=\overline{P M T^{\prime}}-\frac{Q \sum_{k=0}^{\omega-x-1} k e^{-\delta k+\frac{1}{2} \beta^{2} k}{ }_{k} p_{x}}{\sum_{k=0}^{\omega-x-1} e^{-\delta k+\frac{1}{2} \beta^{2} k}{ }_{k} p_{x}}
$$

\section{Actuarial pricing model of double-lives with the stochastic interest rate}

\section{Payment of double-lives pricing model with the stochastic interest rate}

According to lemma 2.3, the couple apply for reverse mortgage. With the stochastic interest rate, the payment of loan amount is given by

$$
\begin{gathered}
{\overline{L S^{\prime}}}_{\overline{x y}}=E(L S(t))=E_{W} E_{N} E_{T(\overline{x y})}(L S(t))=E_{W} E_{N}\left(\int_{0}^{+\infty} L S(t) f_{T_{(\overline{x y})}}(t) d t\right) \\
=\int_{0}^{+\infty} H_{0}(1-h)^{t}(1+g)^{t} E_{W} E_{N}\left(e^{-\delta t-\beta W(t)-\gamma N(t)}\right) f_{T_{(\overline{x y})}}(t) d t \\
=\int_{0}^{\omega-\min \{x, y\}} H_{0}(1-h)^{t}(1+g)^{t} e^{-\delta t+\frac{1}{2} \beta^{2} t}\left(\frac{p}{1-q e^{-\gamma}}\right)^{\alpha} \\
\cdot\left[{ }_{t} p_{x} \cdot \mu_{x+t}+{ }_{t} p_{y} \cdot \mu_{y+t}-{ }_{t} p_{x} \cdot{ }_{t} p_{y}\left(\mu_{x+t}+\mu_{y+t}\right)\right] d t
\end{gathered}
$$

\section{Annuity pricing model of double-lives with the stochastic interest rate}

After the couple signed a loan contract with financing institutions, they get an equal loan from financing institutions until the borrowers die. The discounted value of the annuity equals to Eq. (10), then the loan amount of annuity is given by

$$
\overline{L S^{\prime}}{ }_{\overline{x y}}=\overline{P M T^{\prime}}{ }_{\overline{x y}} E_{W} E_{N} E_{T(\overline{x y})}\left(\sum_{k=0}^{+\infty} I_{\{T(\overline{x y})>k\}} e^{-R(k)}\right)
$$

According to lemma 2.3, the couple applied for reverse mortgage by annuity. With the stochastic interest rate, the beginning of every year the payment of loan amount is give 


$$
\begin{gathered}
\overline{P M T^{\prime}{ }_{\overline{x y}}}=\frac{\overline{L S^{\prime} \overline{x y}}}{E_{W} E_{N} E_{T(\overline{x y})}\left(\sum_{k=0}^{+\infty} I_{\{T(\overline{x y})>k\}} e^{-R(k)}\right)}=\frac{\overline{L S^{\prime}}{ }_{\overline{x y}}}{\sum_{k=0}^{+\infty}{ }_{k} p_{\overline{x y}} E_{W} E_{N}\left(e^{-\delta k-\beta W(k)-\gamma N(k)}\right)} \\
=\frac{\int_{0}^{\omega-\min \{x, y\}} H_{0}(1-h)^{t}(1+g)^{t} e^{-\delta t+\frac{1}{2} \beta^{2} t}\left[{ }_{t} p_{x} \cdot \mu_{x+t}+{ }_{t} p_{y} \cdot \mu_{y+t}-{ }_{t} p_{x} \cdot{ }_{t} p_{y}\left(\mu_{x+t}+\mu_{y+t}\right)\right] d t}{\sum_{k=0}^{\omega-\min \{x, y\}-1} e^{-\delta k+\frac{1}{2} \beta^{2} k}{ }_{k} p_{\overline{x y}}}
\end{gathered}
$$

\section{Linear increasing annuity pricing model of double-lives with the stochastic interest rate}

If the couple get the loan which is increasing by a fixed amount Q every year, the actuarial present value of annuity should be equal to a total amount of payment of Eq. (10).

$$
\begin{aligned}
\overline{L S^{\prime}} \overline{x y}^{\prime} & =E_{W} E_{N} E_{T(\overline{x y})}\left(\sum_{k=0}^{+\infty}\left(\overline{P M T A^{\prime}} \overline{x y}+k Q\right) I_{\{T(\overline{x y})>k\}} e^{-R(k)}\right) \\
& \left.=E_{W} E_{N} E_{T(\overline{x y})}\left(\sum_{k=0}^{+\infty} \overline{P M T A^{\prime}}{ }_{\overline{x y}} I_{\{T(\bar{x})>k\}} e^{-R(k)}\right)+E_{W} E_{N} E_{T(\overline{x y})} \sum_{k=0}^{+\infty} k Q I_{\{T(\overline{x y})>k\}} e^{-R(k)}\right) \\
& \left.=\overline{P M T A^{\prime}}{ }_{\overline{x y}} E_{W} E_{N} E_{T(\overline{x y})}\left(\sum_{k=0}^{+\infty} I_{\{T(\overline{x y})>k\}} e^{-R(k)}\right)+Q E_{W} E_{N} E_{T(\overline{x y})} \sum_{k=0}^{+\infty} k I_{\{T(\overline{x y})>k\}} e^{-R(k)}\right)
\end{aligned}
$$

According to lemma 2.3, the couple apply for reverse mortgage by linear increasing annuity. With the stochastic interest rate, the beginning of the first year the payment of loan amount is given by

$$
\begin{aligned}
& \overline{P M T A^{\prime}} \overline{x y}=\frac{\left.\overline{L S^{\prime}}{ }_{x y}-Q E_{W} E_{N} E_{T(\overline{x y})} \sum_{k=0}^{+\infty} k I_{\{T(\overline{x y})>k\}} e^{-R(k)}\right)}{E_{W} E_{N} E_{T(\overline{x y})}\left(\sum_{k=0}^{+\infty} I_{\{T(\overline{x y})>k\}} e^{-R(k)}\right)}=\frac{\overline{L S^{\prime}}{ }_{\overline{x y}}-Q \sum_{k=0}^{+\infty}{ }_{k} p_{\overline{x y}} k E_{W} E_{N}\left(e^{-\delta k-\beta W(k)-\gamma N(k)}\right)}{\sum_{k=0}^{+\infty}{ }_{k} p_{\overline{x y}} E_{W} E_{N}\left(e^{-\delta k-\beta W(k)-\gamma N(k)}\right)}
\end{aligned}
$$

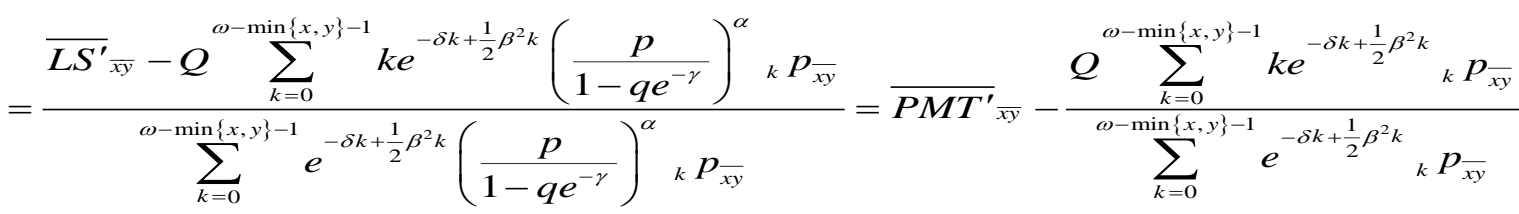

\section{Conclusion}

In this study, the accumulation function model of interest force with a Wiener process and a negative-binomial distribution is proposed as the basis for the reverse mortgage. Using the above model, a lump sum pricing model, annuity pricing model and linear increasing annuity pricing model for single-life and double-lives are provided. All of the models can be improved to solve the problem that the actuarial pricing models of reverse mortgage only could be calculated by the fixed interest rate. These above models provide a guarantee for resolving the risk of the traditional pension funds.

\section{References}

[1] Reed R \& Gibler K. M. The case for reverse mortgage in Australia-applying the USA experience[J]. In 9th Annual Pacific Rim Real Estate Society Conference (2003), p.45-51.

[2] Deokho C \& Ma S. Economic feasibility of reverse mortgage annuity for the elderly housing welfare[J]. Housing Studies Review (2004), p. 175-199.

[3] Tobias Baer \& Isil \& Kanak patel \& Ricardo Pereira \& Sung Jin Yoo. Pricing of reverse mortgage on forward house sale[J]. Proceedings of the 9th Joint Conference on Information Sciences (2006), p.45-90.

[4] Hicks D. Reverse mortgages: a novel tool for financing long-term health care needs[J]. Caring: National Association for Home Care magazine (2005), p. 46-47, 2005.

[5] Bardhan A: Valuing mortgage insurance contracts in emerging market economics to Journal of Real Estate Finance and Economics (2006), p. 9-20. 